\title{
A Preliminary Study for Translation and Validation of the Korean Version of The Cognitive, Affective, and Somatic Empathy Scale in Young Adults
}

\author{
Yeo Eun Park', Ho-Kyoung Yoon², So Young Kim³ ${ }^{3}$ John Williamson ${ }^{4}$, \\ Christian Wallraven ${ }^{1}$, and June Kang ${ }^{1,5} \bowtie$ \\ ${ }^{1}$ Department of Brain and Cognitive Engineering, Korea University, Seoul, Republic of Korea \\ 2Department of Psychiatry, College of Medicine, Korea University, Seoul, Republic of Korea \\ ${ }^{3}$ Department of Psychology, Catholic University, Seoul, Republic of Korea \\ ${ }^{4}$ College of Humanities, Seokyeong University, Seoul, Republic of Korea \\ ${ }^{5}$ Empathy Research Institute, Seoul, Republic of Korea
}

Objective The cognitive, Affective, and Somatic Empathy Scale (CASES) suggests novel three components structure of empathy. Although CASES developed to assess the trait empathy of both children and adult, the validation in adult sample is not yet conducted. This study developed Korean version of CASES (CASES-K) and examined the psychometric properties in young adult for first time.

Methods Confirmatory factor analysis was conducted to test whether the factor structure suggested in initial development study is valid in Korean young adult population. 350 young adults participated, and 44 of them also answered to other empathy scales for validation. The construct validity was ascertained through a series of correlational analysis with widely used trait empathy scales.

Results The result of confirmatory factor analysis supports the three-factor model of empathy consists of affective, cognitive and somatic facets. The construct validity was ascertained through a series of correlational analysis with widely used trait empathy scales. The result of correlational analysis supports the validity of CASES-K, and especially the separation of somatic empathy from affective empathy. Conclusion The current study provides the first evidence of the validity and utility of the CASES-K in young adult population. The result also supports the commonality of three factor structure of empathy in both East Asian and Caucasian, from the children to adults.

Psychiatry Investig 2019;16(9):671-678

Key Words Empathy, Cognitive empathy, Affective empathy, Somatic empathy.

\section{INTRODUCTION}

There is an increasing number of studies concerned with the individual variance of empathy, its neural correlate and consequences like altruistic behavior or conversely, antisocial behavior. Since the quantification of trait empathy is majorly depending on self-report data, a reliable and valid scale is needed to conduct elaborate studies based on individual difference of trait empathy.

Received: April 4, 2019 Revised: May 15, 2019

Accepted: June 25, 2019

$\triangle$ Correspondence: June Kang, $\mathrm{PhD}$

Department of Cognitive and Brain Engineering, Korea University, 145 Anamro, Seongbuk-gu, Seoul 02841, Republic of Korea

Tel: +82-2-6401-0199, Fax: +82-2-6280-1626,

E-mail: june.kang@empathy-research.org

(c) This is an Open Access article distributed under the terms of the Creative Commons Attribution Non-Commercial License (https://creativecommons.org/licenses/by$\mathrm{nc} / 4.0$ ) which permits unrestricted non-commercial use, distribution, and reproduction in any medium, provided the original work is properly cited.
The most of widely used scales are developed based on the concept of multifaceted empathy, which suggests that empathy consists of several separate (but correlated) factors. Most of them divided the empathy into two broad facets, emotional (affective) empathy and cognitive empathy. ${ }^{1-3}$ Cognitive empathy is the ability to aware others' mental state, includes active imagination and putting oneself to other's position. Emotional (affective) empathy can be defined as the ability to experience the perceived emotional state of others, and usually includes the visceral reaction to other's emotional states. Emerging evidences support the orthogonality of those two facets of empathy come from functional neuroimaging studies, ${ }^{4,5}$ lesion studies lesion studies, ${ }^{6,7}$ and studies on psychiatric patients. $^{8-10}$

However, several studies suggested three-component model of empathy. ${ }^{11,12}$ For example, Blair ${ }^{11}$ suggested that empathy is separable into cognitive, affective, and motor em- 
pathy. Motor empathy, also known as, somatic empathy can be defined as "the tendency to automatically mimic and synchronize facial expressions, vocalizations, postures, and movements with those of another person."1 ${ }^{11}$ Following Hatfield and colleagues' conceptualization of emotional contagion, ${ }^{13}$ Blair ${ }^{11}$ argues that somatic(motor) empathy serve as a vehicle for emotional contagion.

Somatic (motor) empathy is usually considered as a part of emotional empathy, but there are several good reasons for teasing apart the somatic (motor) empathy from emotional empathy. First, there are evidences that observing others' emotional expression causes motor mimicry without explicit emotional experience. For example, $30 \mathrm{~ms}$ subliminal exposure of others' facial expression is not sufficient to elicit explicit emotional response, but distinctive facial mimicry was observed without the conscious perception of the stimuli. ${ }^{14}$ Second, interconnected but different neural systems seem to underly motor empathy and emotional empathy processes. Carr and colleagues ${ }^{15}$ suggested the pathway across human mirror neuron system to limbic system through anterior insula as a key network of emotional empathy. Although human mirroring network including inferior frontal and superior temporal cortices seem to deeply involve in empathy process, ${ }^{16}$ it also mediates mere intentional or communicative movements without emotional content. ${ }^{17}$ Third, for the more primitive forms of motor resonance described above, they are not followed by vicarious emotional experience, but related to individual variance of trait empathy. For instance, the degree of automatic mimicry to others' emotional expression measured by facial electromyography correlates with trait emotional empathy. ${ }^{18,19}$

In attempt to address the necessity of consider somatic facet separate from affective facet of empathy, Raine and $\mathrm{Chen}^{20}$ developed a novel self-report tool, Cognitive, Affective, and Somatic Empathy Scales (CASES). CASES shown high validity in the previous studies. The initial development study recruited 428 children in USA (11-12 years, $79.9 \%$ African American, $12.4 \%$ Caucasian). ${ }^{20}$ In initial study, the internal consistency for the total score was 0.91 , and the internal reliability for the three facets are $(0.78-0.81) .^{20} \mathrm{Liu}$ and colleagues $^{21}$ developed a Chinese version of CASES. The validation study was conducted 860 Chinese children (mean age 11.54) shown high (0.92) internal constancy as a whole scale and $0.79-86$ for three facets. ${ }^{21}$

The current study hypothesized that, cognitive empathy of CASES- $\mathrm{K}$ will correlate with the subsets of other scales reflecting cognitive facet of empathy [Perspective Taking (PT) and Fantasy (FS) of Interpersonal Reactivity Index (IRI), Cognitive Empathy (CE) of Empathy Quotient (EQ)], and affective and somatic empathy will correlate with the subsets reflecting affective facet of empathy [Empathic Concern (EC) and Personal Distress (PD) of IRI, Emotional Empathy (EE) of EQ, and the total scores and subsets of Emotional Contagion Scale (ECS)]. Also, the study tested the hypothesis that three factor model of empathy will show better fit compare to one factor or two factor models in Confirmatory Factor Analysis (CFA).

\section{METHODS}

\section{Participants}

Total 350 participants were recruited from Korea University via online advertisement. 2 samples were excluded due to incomplete response. Among 348 participants included in the study, $172(49.5 \%$, mean age $=22.74)$ were female and 176 $(50.5 \%$, mean age $=21.51)$ was male. 44 participants among them $(50 \%$ was male, mean age $=22.66)$ answered additional trait empathy questionnaires (IRI, EQ, and ECS) to assess construct validity of CASES-K. The study was approved by IRB of Korea University (1040548-KU-IRB-15-26-A-2).

\section{Translation}

Original version of CASES was developed using $118 \mathrm{em}-$ pathy questions. ${ }^{20}$ The final version includes 30 items and intended to produce a three-factor model reflects cognitive, affective and somatic facet of empathy, and two-factor model consists of positive and negative valence. The original author approved the translation and validation of Korean version.

The translation procedure followed the standard translation and back-translation procedure suggested by Brislin. ${ }^{22}$ The authors translated the questions from English to Korean. The monolingual Korean reviewed and corrected the translated Korean version for ambiguous or unnatural expressions. Then the Korean version back-translated into English and reviewed by the original authors to find any discrepancies or inconsistencies. Mistranslations were corrected through second back-translation.

\section{Measures}

\section{CASES-K}

30 items CASES-K translated as above procedure was used.

\section{Korean Version of Interpersonal Reactivity Index}

Interpersonal Reactivity Index (IRI) is one of the most widely used self-report measure of trait empathy. IRI consists of 28 items designed to assess empathic trait, which can be divided in four components (PT, FS, EC, PD), suggested by Davis. ${ }^{2} \mathrm{PT}$ and FS considered as cognitive facet, and EC and 
PD are regarded as affective facet of empathy. The Korean version of IRI was translated and validated by Kang and colleagues. ${ }^{23}$ The Cronbach's alpha of the Korean version was 0.80 in validation study ${ }^{23}$ and 0.74 in current study.

\section{Korean Version of Empathy Quotient}

EQ was first developed to assess the individual variance of empathic trait as a spectrum across individuals with highfunction autism or Asperger to highly empathic ones. ${ }^{1}$ Including 20 filter items, EQ consists of 60 four-point Likertstyle questions. EQ was translated and validated in Korean population by Kim and Lee. ${ }^{24}$ The Cronbach's alpha was 0.92 in the initial development study conducted on 197 adult sample. The Cronbach's alpha in Korean validation study was 0.78 in validation study ${ }^{24}$ and 0.69 in current study.

\section{Korean version of Emotion Contagion Scale (ECS-K)}

Emotion Contagion Scale was developed by Doherty, ${ }^{25}$ based on the concept of emotion contagion. ${ }^{26}$ It assumes that the mimicry generates afferent feedback, and the individual variance of the processes determines the susceptibility to others' emotions. ECS consists of 15 items and contains the response toward five emotions of others. Korean version was translated and validated by Kim and Lee. ${ }^{27}$ The confirmatory factor analysis study reported unidimensional factor structure, ${ }^{28}$ but the result from Korean version validation supported two-factors structure consists in positive and negative valence. The initial study on 226 college students reported Cronbach's alpha as $0.90 .{ }^{25}$ Korean version reported $0.83,{ }^{27}$ and 0.835 in current study.

\section{Statistical analysis}

Statistical analyses were conducted using SPSS (version 24, IBM Corp., Armonk, NY, USA) and lavaan package ${ }^{29}$ in R (version 3.4.2, R Foundation for Statistical Computing, Vienna, Austria). To test internal consistency, Cronbach's alpha was calculated. The correlation with other widely-used trait empathy scales (IRI, EQ) were tested to assess construct validity. Confirmatory factor analysis with maximum likelihood estimation was conducted using lavaan package in R. Total three models suggested in initial development-Three factor (cognitive, affective, somatic), two-factor (positive, negative), one factor (overall empathy)-were tested.

Model fit was assessed using multiple goodness-of-fit indices (RMSEA, SRMR, CFI, and chi-square divided by degrees of freedom), since there is no single index reflects desirable features of model. ${ }^{30}$ First, the root mean square error approximation (RMSEA) was calculated. RMSEA is an estimate of the discrepancy between the model and the data per degree of freedom for the model. RMSEA scores that are closer to 0 are preferred, and models with 0.05 are deemed adequate. ${ }^{31}$ Second, the standardized root mean residual (SRMR) was assessed. SRMR is an absolute index of the discrepancy between reproduced and observed correlations. Similar to RMSEA, lower values are preferred in SRMR, and 0.08 or lower suggests an adequate fitting model. ${ }^{32,33}$ Third, the comparative fit index (CFI), a measure of model fit relative to baseline model, whose variables are entirely controlled. ${ }^{34}$ For CFI, 0.95 or higher is considered as adequate model fit. ${ }^{33}$ Lastly, the chi-square divided by degrees of freedom $\left(\chi^{2} / \mathrm{df}\right)$ was calculated. The value less than 3 was considered as "good" model fit. ${ }^{33}$

To compare the model fit between three suggested models, the $\chi^{2}$ difference test with the Satorra-Bentler scaling correction was used. Significant $\chi^{2}$ difference suggests that the less constrained model fits the data better than the more constrained model, as the model fit is significantly improved after some parameters are allowed to be freely estimated instead of being constrained. ${ }^{35}$

\section{RESULTS}

\section{Descriptive statistics}

The mean score of CASES-K was 76,85 and the standard deviation was 8.54 . The normality of the scale considered satisfactory, as the skewness (total: -0.683 , from -1.283 to -0.507 for subscales) and kurtosis (total: 0.077 , from -0.484 to 1.976 for subscales) are fulfill the normality requirements. ${ }^{36}$

\section{Internal reliability and construct validity}

For the entire scale, the internal validity measured by Cronbach's alpha was 0.89 (Table 1). In three factor model, the internal validities of subscales are all satisfactory $(0.76-0.80)$. In two factor model, the internal validity indices of both positive

Table 1. Descriptive statistics of CASES-K $(\mathrm{N}=348)$

\begin{tabular}{lcccc}
\hline \multicolumn{1}{c}{$\begin{array}{c}\text { Mean } \\
\text { (SD) }\end{array}$} & Skewness & Kurtosis & $\begin{array}{c}\text { Cronbach's } \\
\text { Alpha }\end{array}$ \\
\hline $\begin{array}{l}\text { Single factor model } \\
\text { Total (30) }\end{array}$ 76.85(8.54) & -0.683 & 0.077 & 0.89 \\
$\begin{array}{l}\text { Three factor model } \\
\text { Affective (10) }\end{array}$ & $26.27(3.22)$ & -1.283 & 1.976 & 0.76 \\
Cognitive (10) & $25.86(3.14)$ & -0.597 & -0.484 & 0.80 \\
$\begin{array}{l}\text { Somatic (10) } \\
\text { Two factor model }\end{array}$ & $24.71(3.77)$ & -0.628 & -0.110 & 0.80 \\
Positive (15) & $38.35(4.50)$ & -0.507 & -0.432 & 0.81 \\
Negative (15) & $38.49(4.62)$ & -0.791 & 0.396 & 0.82 \\
\hline
\end{tabular}

CASES-K: Korean version of The Cognitive, Affective, and Somatic Empathy Scale 


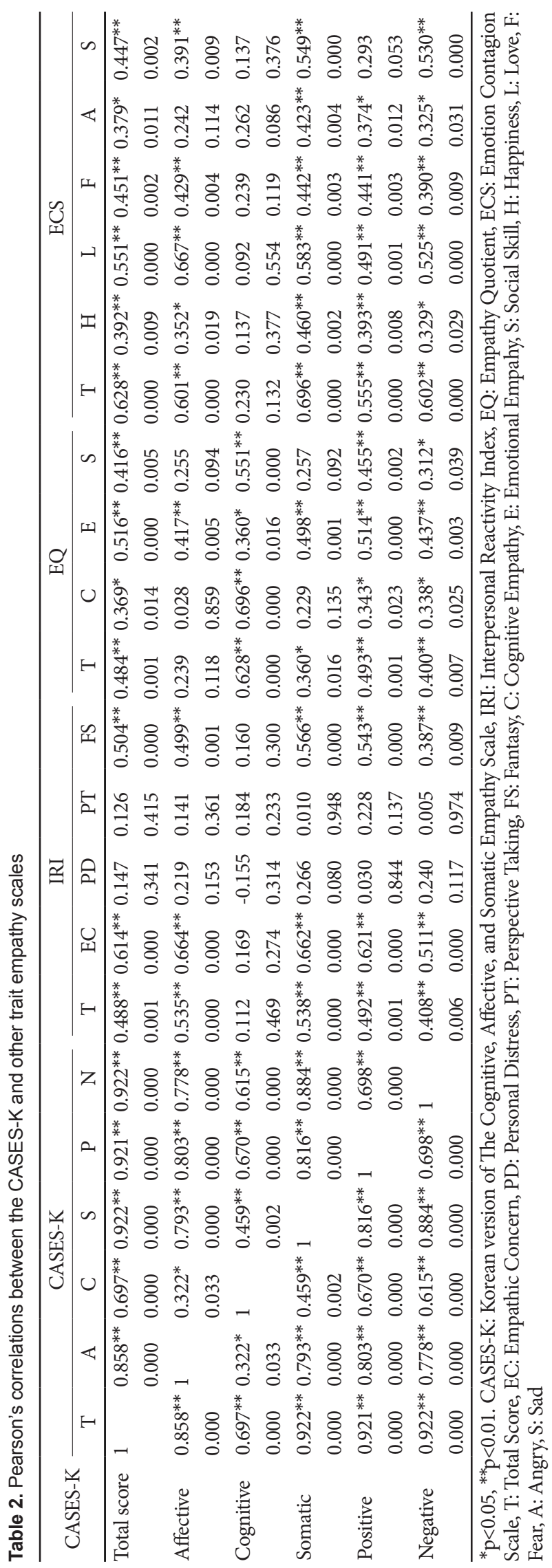

(0.81) and negative (0.82) were also high. The construct validity was assessed by the correlation analysis with other measurements of same or similar psychological constructs like empathy and emotional contagion. Table 2 shows correlations of the CASES-K with scores on other empathy questionnaires.

The CASES-K showed positive correlations with all three empathy scales ( $\mathrm{r}=0.628$ to 0.484$)$. For the three factor model, affective empathy correlates with empathic concern ( $\mathrm{r}=0.664$, $\mathrm{p}<0.001)$ and fantasy $(\mathrm{r}=0.499, \mathrm{p}=0.001)$ subscales of IRI. No significant correlation was found between cognitive empathy and IRI subscales. Somatic empathy correlates with empathic concern $(r=0.662, \mathrm{p}<0.001)$ and fantasy $(\mathrm{r}=0.566, \mathrm{p}<0.001)$. In the correlation analysis with $\mathrm{EQ}$, positive correlation between affective empathy and emotional empathy subscale of EQ ( $r=0.417, p=-0.005)$ was found. Cognitive empathy correlates with all three subscales of EQ ( $r=0.360-0.696, p=0.016^{-}$ $<0.001$ ). Somatic empathy correlates with emotional empathy subscale $(\mathrm{r}=0.498, \mathrm{p}=0.001)$. In the case of correlation with ECS, affective empathy was positively correlates with all subscales of ECS except anger. The correlation with cognitive empathy was not significant at all, and somatic empathy shows significant correlation with all subscales of ECS. For the two factor model, both positive and negative empathy in CASESK positively correlates with EC and FS of IRI. Both subscales shown significant correlations with all subscales in EQ. Positive subscale correlates all ECS subscales except sadness. Negative empathy correlates with all.

\section{Factor structures of CASES-K}

Confirmatory factor analysis using maximum likelihood estimation was conducted to examine three models suggested in the initial development study, ${ }^{20}$ and Chinese validation study (Figure 1). ${ }^{21}$ The model fit indices are presented in Table 3. The first model was a single factor model assumes a general empathy factor. The standardized factor loadings were all statistically significant $(\mathrm{p}<0.001)$ with values ranging from 0.24 to 0.63 (mean $=0.47$ ). The second model was three factor model consists of affective, cognitive and somatic empathy which was the most supported model in previous studies..$^{20,21}$ The standardized factor loading were all statistically significant $(\mathrm{p}<$ 0.001 ) with values ranging from 0.36 to 0.66 (mean $=0.53$ ), which close to suggested common minimum cutoff, $0.40 .{ }^{34}$ The third model consists two factors dividing the empathy phenomenon to ones toward positive and negative valence. The standardized factor loadings were all statistically significant $(\mathrm{p}<0.001)$ with values ranging from 0.24 to 0.69 (mean $=0.48$ ).

The model fit demonstrates that three factor model fulfils the criteria as $\chi^{2} / \mathrm{df}<3.0$, SRMR $<0.08$ indicates the model is adequate but not optimal since the CFI $(<0.90)$ and RMSEA $(<0.06)$ criterion were not met. One factor and two factor 


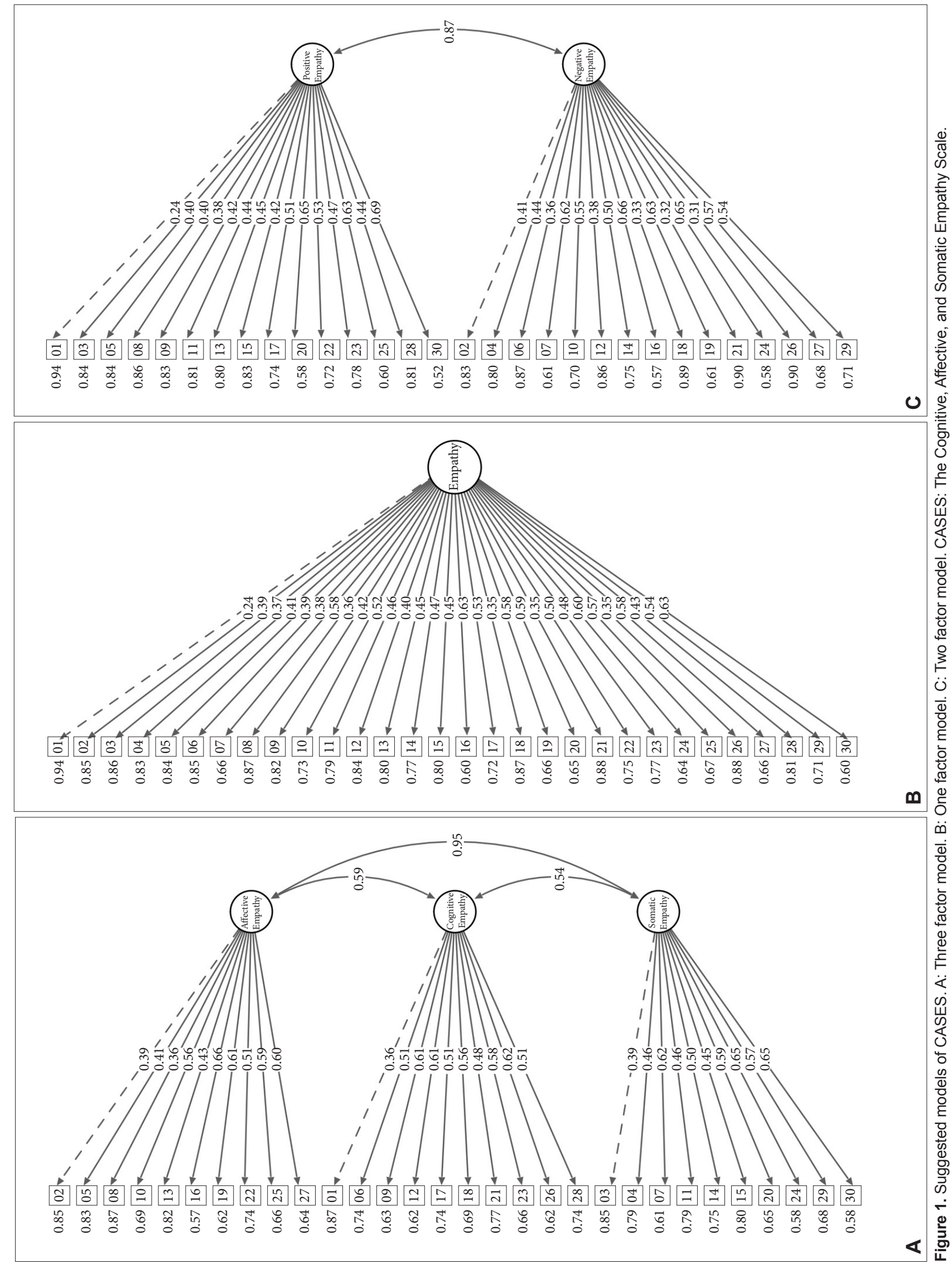


Table 3. Model fit indices of confirmatory factor analysis

\begin{tabular}{lcccccc}
\hline \multicolumn{1}{c}{ Model } & $\chi^{2}$ & $\mathrm{df}$ & $\chi^{2} / \mathrm{df}$ & $\mathrm{CFI}$ & $\mathrm{RMSEA}(95 \% \mathrm{CI})$ & SRMR \\
\hline One-factor model (Empathy) & 1411.137 & 405 & 3.484 & 0.658 & $0.084(0.080-0.089)$ & 0.081 \\
Three-factor model (Affective, Cognitive, \& Somatic) & 1106.866 & 402 & 2.753 & 0.761 & $0.071(0.066-0.076)$ & 0.069 \\
Two-factor model (Positive vs. Negative) & 1369.238 & 404 & 3.389 & 0.672 & $0.083(0.078-0.088)$ & 0.082 \\
\hline
\end{tabular}

CFI: comparative fit index, RMSEA: root mean square error of approximation, SRMR: standardized root mean squared residual, CI: confidence interval

Table 4. Model comparison

\begin{tabular}{lccc}
\hline Model & Satorra-Bentler Scaled $\Delta \chi^{2}$ & $\mathrm{df}$ & $\mathrm{p}$ \\
\hline $\begin{array}{c}\text { Three-factor model vs. } \\
\text { one-factor model }\end{array}$ & 304.271 & 3 & $<0.001$ \\
$\begin{array}{c}\text { Three-factor model vs. } \\
\text { two-factor model }\end{array}$ & 262.372 & 2 & $<0.001$ \\
Two-factor model vs. & & & \\
One-factor model & 41.90 & 1 & $<0.001$ \\
\hline
\end{tabular}

model doesn't fulfil any criteria. The result of model comparison using Satorra-Bentler Scaled $\Delta \chi^{2}$ is described in the Table 4 . Three factor model shown significantly better fit compare to one-factor model $\left[\Delta \chi^{2}(3)=304.271, p<0.001\right]$ and Two-factor model $\left[\Delta \chi^{2}(2)=262.372, \mathrm{p}<0.001\right]$.

\section{DISCUSSION}

The goal of current study was to develop the Korean version of CASES and assess its validity and reliability in Korean young adult. The study showed that the expected three-factor structure is replicated in Korean young adult sample. As the first application of CASES on adult, the results also established the usefulness of CASES in adult through demonstrating the acceptable reliability index and good construct validity.

Construct validity was ascertained by confirming several predicted relationships with other widely used scales of trait empathy. As expected, the affective empathy correlates with empathic concern in IRI. Interestingly, the correlation was not significant with personal distress, which measures the degree of "self-oriented feelings of personal anxiety and unease in intense interpersonal settings." Unexpected positive correlation with fantasy subscale was also observed. The result may reflect the ambiguous property of some items of fantasy subscale of IRI like "I really get involved with the feelings of the characters in a novel," which cannot be conclude to belong to cognitive or affective empathy. In the correlation analysis with EQ, the affective empathy solely correlates with emotional empathy in EQ, and somatic empathy does so. However, Cognitive empathy correlates with all emotional, cognitive and somatic empathy in EQ. As expected, none of the correlation between cognitive empathy and ECS subscales were significant. In contrast, all subscales of ECS reflecting emotional contagion tendency toward five emotions showed positive correlation with both affective and somatic empathy. The result indicates newly derived somatic empathy factor reflects certain aspect of empathy, different from cognitive facet, although how those two facets are different each other is still unclear.

The result in two factor model (positive and negative empathy) is rather inconsistent. Among the other scales of trait empathy used in the current study, ECS was the only scale measured positive (Happiness, Love) and negative (Fear, Anger, Sadness) emotions. Positive empathy of CASES-K was positively correlated with all emotions except sadness ( $\mathrm{p}=0.053)$, but the p-value for sadness was also marginal. Negative empathy of CASES-K was positively correlated with all emotions in ECS. Considering the tendency to being empathic toward positive and negative emotions are generally highly correlates, ${ }^{37}$ and share the neural correlates, ${ }^{38}$ the current finding indicates the dissociation of empathy toward two emotions via trait empathy is not supported.

The results of confirmatory factor analysis support the three-factor model as the most stable one with satisfactory loadings, which consistent with the initial development of English version, ${ }^{20}$ and the validation study of Chinese version. ${ }^{21}$ Nevertheless, taken together with the correlation analysis, two factor model and one factor model are hardly supported.

In comparison to the previous two studies conducted on children, ${ }^{20,21}$ the model fits are not good as ones of those studies. All the models of initial study reported acceptable range of CFI values (0.89-0.84), and RMSEA index (0.43$0.52),{ }^{20}$ and the Chinese validation study reported similar CFI (0.88-0.86), but higher RMSEA $(0.53-0.57) .{ }^{21}$ Since RMSEA decreases when sample size increases, ${ }^{39}$ relatively small sample size may affect the higher RMSEA value of current study. The result is partially due to the items with relatively low factor loading is not excluded in current study. Many previous studies translated and validate the scales in Korean excluded the items with low factor loading. It helps to improve model fit, but make the comparison with the data collected from other languages.

The current study demonstrated the potential of CASES-K as novel tool for assessing individual variance of trait empathy among adult. Similar to the studies conducted on child- 
rens, three factor model shown the best fit among the suggested models. The result supports the cross-cultural and cross-age generalizability of the three factor structure of empathy, which emphasizes the peculiarity of somatic facet of empathy. However, as mentioned above, model fits of current study are not good as ones from those studies.

Considering the interest in the individual difference of trait empathy and their effect on mental i.e., ${ }^{40}$ and physical health i.e., ${ }^{41}$ valid scale that can adequately assess each facet of empathy is very important. Especially, the CASES-K is expected to fulfill the needs to separately consider the somatic aspects from affective facet of empathy in future neuroscientific and psychophysiological studies.

\section{Limitations}

Although current study indicates the potential of CASES-K, this study included only young adult group recruited from university. To extend the usability of the scale, future studies including wider age range and socioeconomic status is needed.

Importantly, the distinct characteristic of CASES is considering somatic empathy separately from emotional empathy. To ensure construct validity of somatic empathy of CASES, it should be tested, whether the somatic response toward others' emotional state is related to somatic empathy of CASES, and the relationship is reasonably orthogonal to ones with emotional empathy of CASES.

\section{Conclusions}

Current study is the first psychometrical validation of CASES in young adult population, and indicates the possible usage of CASES-K as a valid measurement of trait empathy. The result of confirmatory factor analysis supported the three-factor structure suggested in initial development study of English version. ${ }^{20}$ It indicates the three-factor structure is valid across culture ${ }^{21}$ and age. As an instrument assess all three forms of empathy, ${ }^{12}$ CASES-K could be used for advancing the neuroscientific and psychiatric research on empathy and its deficit as a valid index of trait empathy.

\section{Acknowledgments}

This research was supported by NRF grants NRF-2018R1D1A1B07048820 and 2017M3C7A1041824.

\section{Conflicts of Interest}

The authors have no potential conflicts of interest to disclose.

\section{Author Contributions}

Conceptualization: Yeo Eun Park, June Kang. Data curation: Yeo Eun Park, So Young Kim, John Williamson. Formal analysis: Yeo Eun Park, June Kang. Funding acquisition: June Kang. Investigation: Yeo Eun Park. Methodology: June Kang. Project administration: June Kang. Resources: June Kang, So Young Kim. Software: June Kang. Supervision: June Kang. Validation: June Kang, So Young Kim. Visualization: June Kang. Writing- original draft: Yeo Eun Park, June Kang. Writing—review \& editing: June Kang, Ho-Kyoung Yoon.

\section{ORCID iDs}

\section{June Kang}

Yeo Eun Park

https://orcid.org/0000-0003-3625-0856

https://orcid.org/0000-0001-9414-1442

\section{REFERENCES}

1. Baron-Cohen S, Wheelwright S. The empathy quotient: an investigation of adults with Asperger syndrome or high functioning autism, and normal sex differences. J Autism Dev Disord 2004;34:163-175.

2. Davis MH. Measuring individual differences in empathy: evidence for a multidimensional approach. J Pers Soc Psychol 1983;44:113-126.

3. Jolliffe D, Farrington DP. Development and validation of the Basic empathy Scale. J Adolesc 2006;29:589-611.

4. Nummenmaa L, Hirvonen J, Parkkola R, Hietanen JK. Is emotional contagion special? An fMRI study on neural systems for affective and cognitive empathy. Neuroimage 2008;43:571-580.

5. Völlm BA, Taylor AN, Richardson P, Corcoran R, Stirling J, McKie S, et al. Neuronal correlates of theory of mind and empathy: a functional magnetic resonance imaging study in a nonverbal task. Neuroimage 2006;29:90-98.

6. de Sousa A, McDonald S, Rushby J. Changes in emotional empathy, affective responsivity, and behavior following severe traumatic brain injury. J Clin Exp Neuropsychol 2012;34:606-623.

7. Shamay-Tsoory SG, Aharon-Peretz J, Perry D. Two systems for empathy: a double dissociation between emotional and cognitive empathy in inferior frontal gyrus versus ventromedial prefrontal lesions. Brain 2009;132:617-627.

8. Dziobek I, Rogers K, Fleck S, Bahnemann M, Heekeren HR, Wolf OT, et al. Dissociation of cognitive and emotional empathy in adults with Asperger syndrome using the Multifaceted Empathy Test (MET). J Autism Dev Disord 2008;38:464-473.

9. Harari H, Shamay-Tsoory SG, Ravid M, Levkovitz Y. Double dissociation between cognitive and affective empathy in borderline personality disorder. Psychiatry Res 2010; 175: 277-279.

10. Maurage P, Grynberg D, Noël X, Joassin F, Philippot P, Hanak C, et al. Dissociation between affective and cognitive empathy in alcoholism: a specific deficit for the emotional dimension. Alcohol Clin Exp Res 2011;35:1662-1668.

11. Blair RJ. Responding to the emotions of others: dissociating forms of empathy through the study of typical and psychiatric populations. Conscious Cogn 2005;14:698-718.

12. Van der Graaff J, Meeus W, de Wied M, van Boxtel A, van Lier PA, Koot HM, et al. Motor, affective and cognitive empathy in adolescence: Interrelations between facial electromyography and self-reported trait and state measures. Cogn Emot 2016;30:745-761.

13. Hatfield E, Cacioppo JT, Rapson RL. Emotional contagion. Curr Direct Psychol Sci 1993;2:96-100.

14. Dimberg U, Thunberg M, Elmehed K. Unconscious facial reactions to emotional facial expressions. Psychol Sci 2000;11:86-89.

15. Carr L, Iacoboni M, Dubeau MC, Mazziotta JC, Lenzi GL. Neural mechanisms of empathy in humans: a relay from neural systems for imitation to limbic areas. Proc Natl Acad Sci U S A 2003;100:5497-5502.

16. Leslie KR, Johnson-Frey SH, Grafton ST. Functional imaging of face and hand imitation: towards a motor theory of empathy. Neuroimage 2004;21:601-607.

17. Montgomery KJ, Isenberg N, Haxby JV. Communicative hand gestures and object-directed hand movements activated the mirror neuron system. Soc Cogn Affect Neurosci 2007;2:114-122.

18. Sonnby-Borgström M. Automatic mimicry reactions as related to differences in emotional empathy. Scand J Psychol 2002;43:433-443.

19. Dimberg U, Andréasson P, Thunberg M. Emotional empathy and facial reactions to facial expressions. J Psychophysiol 2011;25:26-31. 
20. Raine A, Chen FR. The Cognitive, Affective, and Somatic Empathy Scales (CASES) for children. J Clin Child Adolesc Psychol 2018;47:24-37.

21. Liu JH, Qiao X, Dong FH, Raine A. The Chinese version of the cognitive, affective, and somatic empathy scale for children: Validation, gender invariance and associated factors. Plos One 2018;13:e0195268.

22. Brislin RW. The Wording and Translation of Research Instruments. In: Lonner WJ, Berry JW, Editors. Field Methods in Cross-cultural Research. Beverley Hills, CA: Sage, 1986, p.137-164.

23. Kang I, Kee SW, Kim SE, Jeong BS, Hwang JH, Song JE, et al. Reliability and validity of the Korean-version of Interpersonal Reactivity Index. J Korean Neuropsychiatr Assoc 2009;48:352-358.

24. Kim J, Lee SJ. Reliability and validity of the Korean version of the empathy quotient scale. Psychiatry Investig 2010;7:24-30.

25. Doherty RW. The emotional contagion scale: A measure of individual differences. J Nonverb Behav 1997;21:131-154.

26. Hatfield E, Cacioppo JT, Rapson RL. Emotional contagion. Curr Direct Psychol Sci 1993;2:96-100.

27. Kim MA, Lee JY. Development and Validation of the Korean version of the Emotional Contagion Scale. Korean J Counsel Psychother 2015;27:305-325.

28. Rueff-Lopes R, Caetano A. The emotional contagion scale: factor structure and psychometric properties in a Portuguese sample. Psychol Rep 2012;111:898-904.

29. Oberski D. Iavaan. survey: an R package for complex survey analysis of structural equation models. J Stat Software 2014;57:1-27.

30. Heubeck BG, Neill JT. Confirmatory factor analysis and reliability of the Mental Health Inventory for Australian adolescents. Psychol Rep 2000;87:431-440.

31. Kenny DA, McCoach DB. Effect of the number of variables on measures of fit in structural equation modeling. Struct Equat Modeling
2003;10:333-351.

32. Hu LT, Bentler PM. Cutoff criteria for fit indexes in covariance structure analysis: conventional criteria versus new alternatives. Struct Equat Modeling 1999;6:1-55.

33. Kline RB. Structural equation modeling. 2nd Edition. New York: Guilford Press;2005.

34. Bentler PM. Comparative fit indexes in structural models. Psychol Bull 1990;107:238-246.

35. Schermelleh-Engel K, Moosbrugger H, Müller H. Evaluating the fit of structural equation models: tests of significance and descriptive goodness-of-fit measures. Methods Psychol Res Online 2003;8:23-74.

36. Finney S, Distefano C. Nonnormal and Categorical Data in Structural Equation Modeling. In: Hancock G, Mueller R, Editors. Structural Equation Modeling: A Second Course. Greenwich, CT: Information Age Publishing; 2006.

37. Mehrabian A, Epstein N. A measure of emotional empathy 1. J Pers 1972;40:525-543.

38. Morelli SA, Rameson LT, Lieberman MD. The neural components of empathy: predicting daily prosocial behavior. Soc Cogn Affect Neurosci 2012;9:39-47.

39. Rigdon EE. CFI versus RMSEA: a comparison of two fit indexes for structural equation modeling. Structural Equation Modeling: A Multidisciplinary Journal 1996;3:369-379.

40. Beadle JN, Keady B, Brown V, Tranel D, Paradiso S. Trait empathy as a predictor of individual differences in perceived loneliness. Psychol Rep 2012;110:3-15.

41. Ferguson E. Personality is of central concern to understand health: towards a theoretical model for health psychology. Health Psychol Rev 2013;7:S32-S70 